\title{
Currents in the compressible and incompressible regions of the two-dimensional electron gas
}

\author{
Michael R. Geller and Giovanni Vignale \\ Department of Physics \\ University of Missouri \\ Columbia, Missouri 65211
}

\begin{abstract}
We derive a general expression for the low-temperature current distribution in a two-dimensional electron gas, subjected to a perpendicular magnetic field and in a confining potential that varies slowly on the scale of the magnetic length $\ell$. The analysis is based on a self-consistent one-electron description, such as the Hartree or standard Kohn-Sham equations. Our expression, which correctly describes the current distribution on scales larger than $\ell$, has two components: One is an "edge current" which is proportional to the local density gradient and the other is a "bulk current" which is proportional to the gradient of the confining potential. The direction of these currents generally display a striking alternating pattern. In a compressible region at the edge of the $n$th Landau level, the magnitude of the edge current is simply $\mathbf{j}=-e \omega_{\mathrm{c}} \ell^{2}\left(n+\frac{1}{2}\right) \nabla \rho \times \mathbf{e}_{z}$ where $\omega_{\mathrm{c}}$ is the cyclotron frequency and $\rho$ is the electron density. The bulk component, a Hall current, dominates in the incompressible regions. In the ideal case of perfect compressibility and incompressibility, only one type of current contributes to a given region, and the integrated currents in these regions are universal, independent of the widths, positions, and geometry of the regions. The integrated current in the $n$th edge channel is $\left(n+\frac{1}{2}\right) e \omega_{\mathrm{c}} / 2 \pi$, whereas in an incompressible strip with integral filling factor $\nu$ it is $\nu e \omega_{\mathrm{c}} / 2 \pi$ with the opposite sign.

PACS numbers: 73.20.Dx, 73.40.-c, 73.40.Hm, 73.50.-h
\end{abstract}




\section{INTRODUCTION}

In the past several years, there has been tremendous interest in the low-temperature properties of a two-dimensional (2D) electron gas in a strong magnetic field. The most common experimental realization of this system is at a modulation-doped semiconductor heterojunction, grown by molecular beam epitaxy. One important question, now receiving much attention, is the distribution of current when the system is subjected to a confining potential, or to an applied voltage, or to both. Knowledge of the equilibrium and nonequilibrium current distributions is important for understanding the quantum Hall effect, the electronic properties of low-dimensional semiconductor nanostructures such as quantum dots or quantum wires in the presence of a magnetic field, and for understanding mesoscopic transport in general. The current distribution can also be used to calculate the magnetic properties (for example, the orbital magnetization) of a confined 2D electron gas.

Two types of methods are commonly used to obtain a confined 2D electron gas. Lithographic methods result in the well-known etched structures consisting of a patterned region of the $2 \mathrm{D}$ electron gas along with its compensating positively charged donors. The second method produces a confining potential via one or more evaporated metal gates. In both cases, the actual confining potential is the sum of the potential from the remote donors and gates, plus the self-consistent electrostatic potential (and possibly the exchange-correlation scalar potential) of the electrons. Typical electron sheet densities in the modulation-doped $2 \mathrm{D}$ electron gas vary from $10^{11}$ to $10^{12} \mathrm{~cm}^{-2}$. The most interesting effects of an applied magnetic field then occur at field strengths ranging from 1 to 10 Tesla, where only a few Landau levels are occupied. At these high field strengths, the magnetic length, ranging from 50 to a few hundred $\AA$, is small compared with the length scale over which the confining potential changes by $\hbar \omega_{c}$, where $\omega_{\mathrm{c}}$ is the cyclotron frequency. In this sense, the 
confining potential is slowly varying.

Vignale and Skudlarski ${ }^{1}$ have recently used current-density functional theory ${ }^{2}$ to derive an exact formal relation between the ground-state current and density distributions of a three-dimensional interacting electron gas in the presence of a magnetic field. In the local density approximation (LDA), valid when the density varies slowly on the scale of the magnetic length, they obtain an explicit formula for the current in terms of the density gradient with a coefficient of proportionality involving thermodynamic quantities for a uniform electron gas. However, the application of this LDA result to the 2D electron gas is complicated by the presence of incompressible regions, where the density gradient vanishes and the coefficient diverges. Furthermore, in the 2D electron gas, the density may change from one integral filling factor to another over a single magnetic length, invalidating the a priori application of the LDA. This has motivated us to reexamine the relation between current and density in two dimensions, without using the LDA.

Another motivation for this work is to study the equilibrium currents in edge channels. ${ }^{3-7}$ In a recent paper, Chklovskii et al. ${ }^{5}$ have calculated the classical electrostatic potential and density of a gate-confined 2D electron gas. They show that the electrostatic potential consists of a series of wide steps of height $\hbar \omega_{\mathrm{c}}$. In contrast to a naive single-electron picture, there are wide compressible regions where the density gradually changes from one integral filling factor to another, the so-called edge channels, and narrow incompressible regions of integral filling factor. This type of bahavior had been previously noted by McEuen et $a l .{ }^{6}$ in the context of quantum dots. The classical electrostatic analysis of Chklovskii et al. has also been extended to narrow gate-confined channels ${ }^{7}$ and to quantum dots. ${ }^{8}$ The electrostatics of edge channels in mesa-etched samples has been studied by Gelfand and Halperin, ${ }^{9}$ and considerable effort has been recently devoted to extending the classical electrostatic treatment to self-consistent Hartree and Hartree-Fock approximations. ${ }^{9-13}$ A related question is the transition between sharp and smooth density distributions as the 
slope of the confining potential is changed. ${ }^{13,14}$

Prior to the recent work on edge channels, considerable progress had already been made in understanding the distribution of current in the quantum Hall regime. In one of the early papers on this subject, MacDonald et al. ${ }^{15}$ used the localized nature of the Landau states to show that the ground-state current density, directed in the $y$ direction along a Hall bar whose confining potential $V(x)$ varies in the $x$ direction only, is simply proportional to $V^{\prime}(x)$ in the interior of the Hall bar. Several authors ${ }^{15-20}$ have calculated nonequilibrium density and current distributions in particular confining potentials, and the correct description of the nonequilibrium steady state is now a problem of great interest. $^{18-23}$ In particular, Thouless has emphasized the importance of nonequilibrium bulk currents that are induced by edge-charge redistribution. ${ }^{20}$

In this paper, we derive the low-temperature $\left(k_{\mathrm{B}} T<<\hbar \omega_{\mathrm{c}}\right)$ density and current distributions for a high-mobility spin-polarized 2D electron gas in an arbitrary confining potential $V(\mathbf{r})$ and uniform magnetic field $\mathbf{B}=B \mathbf{e}_{z}$, assuming only that the potential varies slowly on the scale of the magnetic length $\ell \equiv(\hbar c / e B)^{\frac{1}{2}}$. As stated above, the confining potential is assumed to consist of an external potential, from remote donor centers and gates, and a self-consistent Hartree potential or Hartree plus exchange-correlation scalar potential. A study of exchange and correlation effects, based on current-density functional theory, ${ }^{2}$ shall be published elsewhere. We now briefly summarize our results.

We shall show that the low-temperature equilibrium current density (for electrons of charge $-e$ ) may be written as $\mathbf{j}=\mathbf{j}_{\text {edge }}+\mathbf{j}_{\text {bulk }}$, where

$$
\mathbf{j}_{\text {edge }} \equiv-e \omega_{\mathrm{c}} \ell^{2} \sum_{n=0}^{\infty}\left(n+\frac{1}{2}\right) \nabla \rho_{n}(\mathbf{r}) \times \mathbf{e}_{z}
$$

and

$$
\mathbf{j}_{\text {bulk }} \equiv-\frac{e}{m \omega_{\mathrm{c}}} \rho(\mathbf{r}) \nabla V(\mathbf{r}) \times \mathbf{e}_{z}
$$


Here $\omega_{\mathrm{c}} \equiv e B / m c$ is the cyclotron frequency. The electron number density, $\rho$, is given by

$$
\rho=\frac{1}{2 \pi \ell^{2}} \sum_{n=0}^{\infty} f\left[\hbar \omega_{\mathrm{c}}\left(n+\frac{1}{2}\right)+V(\mathbf{r})-\mu\right]
$$

where $\mu$ is the chemical potential, $f(\epsilon) \equiv\left(e^{\epsilon / k_{\mathrm{B}} T}+1\right)^{-1}$ is the Fermi distribution function, and $\rho_{n}$ is simply the $n$th term in (1.3). At low temperatures, the electron density is uniform everywhere except near a number of edges, where the density changes by an amount $\left(2 \pi \ell^{2}\right)^{-1}$. These compressible regions, or edge channels, follow lines of constant confining potential. The edge current (1.1) is a sum of nonoverlapping parts; the contribution from the edge of the $n$th Landau level is $\mathbf{j}_{\text {edge }}=-e \omega_{\mathrm{c}} \ell^{2}\left(n+\frac{1}{2}\right) \nabla \rho \times \mathbf{e}_{z}$. In terms of the local electric field $\mathbf{E}=\nabla V / e$, the bulk current (1.2) may also be written as $j_{\text {bulk }}^{i}=\sigma^{i j}(\mathbf{r}) E^{j}(\mathbf{r})$, where $\sigma^{i j}(\mathbf{r})$ is the local Hall conductivity tensor. We shall show that the sign difference in the edge current relative to the bulk current, which follows from the fact that $\nabla \rho$ and $\nabla V$ are antiparallel, leads to striking oscillations in the direction of the current in a confined $2 \mathrm{D}$ electron gas.

The organization of this paper is as follows. In the next section, we construct the single-particle Green's function for the confined electron gas by a gradient expansion in the confining potential. We keep terms corresponding to the local magnitude of the confining potential and its first derivative. The neglect of higher order derivatives results in distributions which are correct on scales larger than $\ell$. We then use this Green's function to compute the equilibrium density and current distributions. In Section III, we compare our results with exact distributions obtained for the case of noninteracting electrons with parabolic confinement. In Section IV, we study three applications. First, we calculate the low-temperature current distribution in a stepped potential characteristic of the selfconsistent Hartree potential for a narrow gate-confined Hall bar, a system well-known to possess alternating strips of compressible and incompressible electron gas. Second, we show that the integrated current in an ideal incompressible strip with integral filling factor 
$\nu$ is $\nu e \omega_{\mathrm{c}} / 2 \pi$, independent of the strip position, geometry, and width. Similarly, the magnitude of the integrated current in an ideal edge channel at the edge of the $n$th Landau level is found to be $\left(n+\frac{1}{2}\right) e \omega_{\mathrm{c}} / 2 \pi$, independent of the channel position, geometry, and width. The directions of these currents display an alternating pattern. As a third application, we show that the total azimuthal current in a quantum dot, as a function of particle number, is quantized in units of $e \omega_{\mathrm{c}} / 4 \pi$. In an appendix we show that our expression for the current density also follows from the long-wavelength limit of the appropriate linear response functions, and we also show that a careful treatment of the divergences in the LDA relation between current and density also leads to our result. The physical origin of the alternating signs of the edge and bulk currents is also explained there. 


\section{EQUILIBRIUM CURRENT DISTRIBUTION}

We shall first obtain the single-particle Green's function for the confined electron gas by the following method: (i) First, a Dyson equation is obtained for the Green's function $G$ of the confined electron gas, in terms of the Green's function $G^{0}$ of the uniform electron gas and the confining potential $V(\mathbf{r})$; (ii) Then the short-ranged nature of $G^{0}$ is used to separate the potential near $\mathbf{r}$ into a local constant potential, $V(\overline{\mathbf{r}})$, and a gradient, $(\mathbf{r}-\overline{\mathbf{r}}) \cdot \nabla V(\overline{\mathbf{r}})$, for some $\overline{\mathbf{r}}$ near $\mathbf{r}$; (iii) Next, the local constant potential terms are summed to all orders, resulting in a Green's function $G^{1}$; (iv) The gradient terms are then treated to first order, resulting in a first order gradient expansion for $G$; (v) Finally, the local direction of the potential gradient is used to find a gauge in which the Green's function takes a particularly simple form, corresponding to an expansion in eigenstates that are localized in the $\nabla V(\overline{\mathbf{r}})$ direction.

As stated above, we consider a 2D electron gas in a uniform magnetic field $\mathbf{B}=B \mathbf{e}_{z}$ and in a slowly varying potential $V(\mathbf{r})$, where $\mathbf{r}=(x, y)$. We assume that the electrons are spin-polarized by the strong magnetic field, and we disregard the resulting constant Zeeman energy. The Hamiltonian may be written as

$$
H=H^{0}+V
$$

where $H^{0} \equiv \frac{1}{2 m}\left(\mathbf{p}+\frac{e}{c} \mathbf{A}\right)^{2}$ is the Hamiltonian for an electron in the presence of the magnetic field alone. In terms of the exact normalized eigenstates $\Psi_{\alpha}$ and eigenvalues $E_{\alpha}$ of $H$, the Green's function for the confined electron gas may be written as

$$
G\left(\mathbf{r}, \mathbf{r}^{\prime}, s\right)=\sum_{\alpha} \frac{\Psi_{\alpha}(\mathbf{r}) \Psi_{\alpha}^{*}\left(\mathbf{r}^{\prime}\right)}{s-E_{\alpha}}
$$

where $s$ is a complex energy variable. Knowledge of the Green's function allows one to determine the equilibrium number density,

$$
\rho(\mathbf{r})=\oint \frac{d s}{2 \pi i} f[s-\mu] G(\mathbf{r}, \mathbf{r}, s),
$$


and current density,

$$
\mathbf{j}(\mathbf{r})=-\frac{e}{m} \oint \frac{d s}{2 \pi i} f[s-\mu] \lim _{\mathbf{r}^{\prime} \rightarrow \mathbf{r}} \operatorname{Re}\left(-i \hbar \nabla+\frac{e}{c} \mathbf{A}\right) G\left(\mathbf{r}, \mathbf{r}^{\prime}, s\right),
$$

at fixed chemical potential $\mu$. The contour in the complex energy plane is to be taken in the positive sense around the poles of $G$ on the real $s$ axis, avoiding the poles of $f$.

The Green's function $G^{0}\left(\mathbf{r}, \mathbf{r}^{\prime}, s\right)$ for the unconfined electron gas may be written as

$$
G^{0}\left(\mathbf{r}, \mathbf{r}^{\prime}, s\right)=\sum_{n} \int_{-\infty}^{\infty} d q \frac{\phi_{n q}(\mathbf{r}) \phi_{n q}^{*}\left(\mathbf{r}^{\prime}\right)}{s-\hbar \omega_{\mathrm{c}}\left(n+\frac{1}{2}\right)}
$$

where the $\phi_{n q}(n=0,1,2, \cdots)$ are the normalized eigenstates of $H^{0}$,

$$
H^{0} \phi_{n q}=\hbar \omega_{\mathrm{c}}\left(n+\frac{1}{2}\right) \phi_{n q}
$$

In the gauge $\mathbf{A}=B x \mathbf{e}_{y}$ these eigenstates are

$$
\phi_{n q}=C_{n} e^{-i q y} e^{-\frac{1}{2}\left(\frac{x}{\ell}-q \ell\right)^{2}} H_{n}\left(\frac{x}{\ell}-q \ell\right),
$$

where the $H_{n}$ are Hermite polynomials, and where $C_{n} \equiv\left(2^{n+1} n ! \pi^{\frac{3}{2}} \ell\right)^{-\frac{1}{2}}$. These states are normalized according to $\int d^{2} r \phi_{n q}^{*} \phi_{n^{\prime} q^{\prime}}=\delta_{n n^{\prime}} \delta\left(q-q^{\prime}\right)$.

The Green's functions $G$ and $G^{0}$ may be related by the Dyson equation

$$
G\left(\mathbf{r}, \mathbf{r}^{\prime}, s\right)=G^{0}\left(\mathbf{r}, \mathbf{r}^{\prime}, s\right)+\int d^{2} r^{\prime \prime} G^{0}\left(\mathbf{r}, \mathbf{r}^{\prime \prime}, s\right) V\left(\mathbf{r}^{\prime \prime}\right) G\left(\mathbf{r}^{\prime \prime}, \mathbf{r}^{\prime}, s\right) .
$$

For large $\left|\mathbf{r}-\mathbf{r}^{\prime}\right|$, the magnitude of the Green's function $G^{0}\left(\mathbf{r}, \mathbf{r}^{\prime}, s\right)$ falls off as a Gaussian $e^{-\frac{1}{4}\left|\mathbf{r}-\mathbf{r}^{\prime}\right|^{2} / \ell^{2}}$, except at its poles. Then

$$
\begin{gathered}
G\left(\mathbf{r}, \mathbf{r}^{\prime}, s\right)=G^{0}\left(\mathbf{r}, \mathbf{r}^{\prime}, s\right) \\
+\int d^{2} r^{\prime \prime} G^{0}\left(\mathbf{r}, \mathbf{r}^{\prime \prime}, s\right)\left[V(\overline{\mathbf{r}})+\left(\mathbf{r}^{\prime \prime}-\overline{\mathbf{r}}\right) \cdot \nabla V(\overline{\mathbf{r}})\right] G\left(\mathbf{r}^{\prime \prime}, \mathbf{r}^{\prime}, s\right),
\end{gathered}
$$

where $\overline{\mathbf{r}}$ is any point near $\mathbf{r}$, and where higher order gradient terms have been neglected. Equation (2.9) is now solved iteratively, keeping all terms containing no gradients and all terms containing one local gradient. This leads to

$$
G\left(\mathbf{r}, \mathbf{r}^{\prime}, s\right)=G^{1}\left(\mathbf{r}, \mathbf{r}^{\prime}, s\right)+\int d^{2} r^{\prime \prime} G^{1}\left(\mathbf{r}, \mathbf{r}^{\prime \prime}, s\right)\left(\mathbf{r}^{\prime \prime}-\overline{\mathbf{r}}\right) \cdot \nabla V(\overline{\mathbf{r}}) G^{1}\left(\mathbf{r}^{\prime \prime}, \mathbf{r}^{\prime}, s\right),
$$


where $G^{1}\left(\mathbf{r}, \mathbf{r}^{\prime}, s\right)$ satisfies

$$
G^{1}\left(\mathbf{r}, \mathbf{r}^{\prime}, s\right)=G^{0}\left(\mathbf{r}, \mathbf{r}^{\prime}, s\right)+V(\overline{\mathbf{r}}) \int d^{2} r^{\prime \prime} G^{0}\left(\mathbf{r}, \mathbf{r}^{\prime \prime}, s\right) G^{1}\left(\mathbf{r}^{\prime \prime}, \mathbf{r}^{\prime}, s\right)
$$

For notational simplicity, the dependence of $G^{1}$ on $\overline{\mathbf{r}}$ has been suppressed. The solution of the integral equation $(2.11)$ is

$$
G^{1}\left(\mathbf{r}, \mathbf{r}^{\prime}, s\right)=G^{0}\left(\mathbf{r}, \mathbf{r}^{\prime}, s-V(\overline{\mathbf{r}})\right)
$$

valid for any $\overline{\mathbf{r}}$ near $\mathbf{r}$. The Green's function $G^{0}$, when renormalized by the local potential $V(\overline{\mathbf{r}})$, simply has its energy argument shifted by $V(\overline{\mathbf{r}})$. Because of the arbitrariness in the choice of $\overline{\mathbf{r}}, G^{1}$ is not unique. However, the effect of a change of $\overline{\mathbf{r}}$ on $G^{1}$ is compensated for by the corresponding change in the second term in (2.10), and the complete Green's function (2.10) is independent of $\overline{\mathbf{r}}$ to first order in the local gradient $\nabla V(\overline{\mathbf{r}})$.

At this point we have obtained a gradient expansion for the Green's function $G$. Unfortunately, the expression (2.10) contains all matrix elements $\left\langle n q|\mathbf{r}| n^{\prime} q^{\prime}\right\rangle$ of $\mathbf{r}$ in the basis (2.7). To circumvent this, we shall perform a gauge transformation, for each $\overline{\mathbf{r}}$, which rotates the direction of the vector potential so that it is perpendicular to the local gradient of the confining potential. The gauge-transformed Green's functions may then be written in terms of eigenfunctions which are localized in the $\nabla V(\overline{\mathbf{r}})$ direction, resulting in a simple closed-form expression for $G$.

To this end, we shall use the slowly varying function $V(\mathbf{r})$ to define a local orthonormal basis $\mathbf{n}_{a}(a=1,2)$ on the $z=0$ plane:

$$
\begin{gathered}
\mathbf{n}_{1}(\mathbf{r}) \equiv \frac{\nabla V(\mathbf{r})}{|\nabla V(\mathbf{r})|} \\
\mathbf{n}_{2}(\mathbf{r}) \equiv \mathbf{e}_{z} \times \mathbf{n}_{1}(\mathbf{r}) .
\end{gathered}
$$

These basis vectors clearly satisfy the orthonormality condition $\mathbf{n}_{a} \cdot \mathbf{n}_{b}=\delta_{a b}$ and are oriented according to $\mathbf{n}_{1} \times \mathbf{n}_{2}=\mathbf{e}_{z}$. A vector potential directed parallel to $\mathbf{n}_{2}(\overline{\mathbf{r}})$, for some 
fixed $\overline{\mathbf{r}}$, and hence directed perpendicular to $\nabla V(\overline{\mathbf{r}})$, is given by

$$
\mathbf{A}^{\prime} \equiv B\left[\mathbf{n}_{1}(\overline{\mathbf{r}}) \cdot \mathbf{r}\right] \mathbf{n}_{2}(\overline{\mathbf{r}})
$$

We suppress the parametric dependence of $\mathbf{A}^{\prime}$ on $\overline{\mathbf{r}}$. This vector potential describes a uniform magnetic field $\left(\nabla \times \mathbf{A}^{\prime}=\mathbf{B}\right)$ and is transverse $\left(\nabla \cdot \mathbf{A}^{\prime}=0\right)$. The normalized eigenstates of $H^{0}$ in this gauge are

$$
\psi_{n q}(\mathbf{r})=C_{n} e^{-i q \eta \ell} e^{-\frac{1}{2}(\xi-q \ell)^{2}} H_{n}(\xi-q \ell)
$$

where $\xi \equiv \mathbf{n}_{1}(\overline{\mathbf{r}}) \cdot \mathbf{r} / \ell$ and $\eta \equiv \mathbf{n}_{2}(\overline{\mathbf{r}}) \cdot \mathbf{r} / \ell$. The dependence of $\xi$ and $\eta$ on $\overline{\mathbf{r}}$ has also been suppressed.

The Green's function $G_{A^{\prime}}$, computed in the gauge $\mathbf{A}^{\prime}=\mathbf{A}+\nabla \Lambda$, is related to the Green's function $G_{A}$ in the original gauge by

$$
G_{A}\left(\mathbf{r}, \mathbf{r}^{\prime}, s\right)=e^{\frac{i e}{\hbar c}\left[\Lambda(\mathbf{r})-\Lambda\left(\mathbf{r}^{\prime}\right)\right]} G_{A^{\prime}}\left(\mathbf{r}, \mathbf{r}^{\prime}, s\right)
$$

This allows one to compute a given matrix element ( $\mathbf{r}$ and $\mathbf{r}^{\prime}$ regarded as matrix indices) of $G_{A}$ by transforming to some gauge $\mathbf{A}^{\prime}$ where $G_{A^{\prime}}$ is simpler. One may choose a different gauge for each matrix element. The generator of the gauge transformation from $\mathbf{A}=B x \mathbf{e}_{y}$ to $\mathbf{A}^{\prime}$ is given by

$$
\nabla \Lambda(\mathbf{r})=B\left[\mathbf{n}_{1}(\overline{\mathbf{r}}) \cdot \mathbf{r}\right] \mathbf{n}_{2}(\overline{\mathbf{r}})-B x \mathbf{e}_{y}
$$

Then we obtain

$$
\begin{gathered}
G_{A}\left(\mathbf{r}, \mathbf{r}^{\prime}, s\right)=e^{\frac{i e}{\hbar c}\left[\Lambda(\mathbf{r})-\Lambda\left(\mathbf{r}^{\prime}\right)\right]\left[\sum_{n} \int_{-\infty}^{\infty} d q \frac{\psi_{n q}(\mathbf{r}) \psi_{n q}^{*}\left(\mathbf{r}^{\prime}\right)}{s-\hbar \omega_{\mathrm{c}}\left(n+\frac{1}{2}\right)-V(\overline{\mathbf{r}})}\right.} \\
\left.+\nabla V(\overline{\mathbf{r}}) \cdot \sum_{n n^{\prime}} \int_{-\infty}^{\infty} d q d q^{\prime} \frac{\left[\left\langle n q|\mathbf{r}| n^{\prime} q^{\prime}\right\rangle-\overline{\mathbf{r}} \delta_{n n^{\prime}} \delta\left(q-q^{\prime}\right)\right] \psi_{n q}(\mathbf{r}) \psi_{n^{\prime} q^{\prime}}^{*}\left(\mathbf{r}^{\prime}\right)}{\left[s-\hbar \omega_{\mathrm{c}}\left(n+\frac{1}{2}\right)-V(\overline{\mathbf{r}})\right]\left[s-\hbar \omega_{\mathrm{c}}\left(n^{\prime}+\frac{1}{2}\right)-V(\overline{\mathbf{r}})\right]}\right],
\end{gathered}
$$

where $\Lambda$ is given by (2.17). The matrix elements $\left\langle n q|\mathbf{r}| n^{\prime} q^{\prime}\right\rangle$ are now in the basis (2.15). Because $\nabla V(\overline{\mathbf{r}})$ points in the $\mathbf{n}_{1}(\overline{\mathbf{r}})$ direction, only the matrix elements of $\mathbf{n}_{1} \cdot \mathbf{r}$ are required. 
In what follows, we shall always choose $\overline{\mathbf{r}}=\mathbf{r}$; the symmetric choice $\overline{\mathbf{r}}=\frac{1}{2}\left(\mathbf{r}+\mathbf{r}^{\prime}\right)$ yields identical results. This concludes our construction of the Green's function for the 2D electron gas in a magnetic field and a slowly varying confining potential.

We now calculate the equilibrium density of the confined electron gas. The first term in (2.18) contributes an amount

$$
\frac{1}{2 \pi \ell^{2}} \sum_{n} f\left[\hbar \omega_{\mathrm{c}}\left(n+\frac{1}{2}\right)+V(\mathbf{r})-\mu\right]
$$

The contribution to the density from the second term in (2.18) vanishes. Therefore, the final result is that stated in (1.3).

We shall calculate the equilibrium current density by expanding $\mathbf{j}(\mathbf{r})$ in the local basis $\mathbf{n}_{a}$,

$$
\mathbf{j}(\mathbf{r})=j_{1}(\mathbf{r}) \mathbf{n}_{1}(\mathbf{r})+j_{2}(\mathbf{r}) \mathbf{n}_{2}(\mathbf{r})
$$

where $j_{a}(\mathbf{r}) \equiv \mathbf{j}(\mathbf{r}) \cdot \mathbf{n}_{a}(\mathbf{r})$. The component of the current density along the local potential gradient is

$$
j_{1}=-\frac{e}{m} \oint \frac{d s}{2 \pi i} f[s-\mu] \lim _{\mathbf{r}^{\prime} \rightarrow \mathbf{r}} \operatorname{Re}\left(-i \hbar \mathbf{n}_{1} \cdot \nabla+\frac{e}{c} \mathbf{n}_{1} \cdot \mathbf{A}\right) G\left(\mathbf{r}, \mathbf{r}^{\prime}, s\right) .
$$

Using (2.18) leads to

$$
j_{1}=-e \omega_{\mathrm{c}} \ell^{2} \frac{e}{\hbar c} \mathbf{n}_{1} \cdot \nabla \Lambda \rho-e \omega_{\mathrm{c}} x \mathbf{n}_{1} \cdot \mathbf{e}_{y} \rho
$$

The first term in (2.22) comes from the $\mathbf{n}_{1} \cdot \nabla$ acting on the exponential in (2.18), and the second term comes from the diamagnetic part of (2.21). There is no contribution from $\mathbf{n}_{1} \cdot \nabla$ acting on $G_{A^{\prime}}$ (the quantity in square brackets) because these derivatives are real. Using (2.17), we see that $j_{1}$ vanishes. The transverse current density is given by

$$
\begin{aligned}
j_{2}= & e \omega_{\mathrm{c}} \ell^{2} \oint \frac{d s}{2 \pi i} f[s-\mu] \operatorname{Re}\left(-\frac{e}{\hbar c} \mathbf{n}_{2} \cdot \nabla \Lambda(\mathbf{r}) G_{A^{\prime}}(\mathbf{r}, \mathbf{r}, s)\right. \\
& \left.+i \lim _{\mathbf{r}^{\prime} \rightarrow \mathbf{r}} \mathbf{n}_{2} \cdot \nabla G_{A^{\prime}}\left(\mathbf{r}, \mathbf{r}^{\prime}, s\right)\right)-\frac{e^{2}}{m c} B x \mathbf{n}_{2} \cdot \mathbf{e}_{y} \rho .
\end{aligned}
$$


A straightforward calculation leads to

$$
\begin{gathered}
j_{2}=-\frac{e^{2} \omega_{\mathrm{c}} \ell^{2}}{\hbar c} \rho \mathbf{n}_{2} \cdot \nabla \Lambda+e \omega_{\mathrm{c}} \ell \xi \rho \\
-e \omega_{\mathrm{c}} \ell^{2} \sum_{n}\left(n+\frac{1}{2}\right)\left|\nabla \rho_{n}\right|+e \omega_{\mathrm{c}} \ell^{2} \frac{|\nabla V|}{\hbar \omega_{\mathrm{c}}} \rho-e \omega_{\mathrm{c}} x \rho \mathbf{n}_{2} \cdot \mathbf{e}_{y},
\end{gathered}
$$

where $\rho_{n} \equiv\left(1 / 2 \pi \ell^{2}\right) f\left[\hbar \omega_{\mathrm{c}}\left(n+\frac{1}{2}\right)+V(\mathbf{r})-\mu\right]$. Finally, after using $(2.17)$, we find

$$
j_{2}=-e \omega_{\mathrm{c}} \ell^{2} \sum_{n}\left(n+\frac{1}{2}\right)\left|\nabla \rho_{n}\right|+e \omega_{\mathrm{c}} \ell^{2} \frac{|\nabla V|}{\hbar \omega_{\mathrm{c}}} \rho .
$$

Note that $\nabla \rho$ is antiparallel to $\nabla V$. Therefore, the equilibrium current density is given by the expression stated in Section I. 


\section{COMPARISON WITH AN EXACTLY SOLVABLE CASE}

In this section, we compare our results to the exact equilibrium density and current distributions of a noninteracting 2D electron gas in a uniform magnetic field $\mathbf{B}=B \mathbf{e}_{z}$ and a parabolic confining potential

$$
V(x)=\frac{1}{2} m \omega_{0}^{2} x^{2} .
$$

The results derived above apply to the case where $\omega_{0}<<\omega_{\mathrm{c}}$, so that the potential is slowly varying over a range of several magnetic lengths. In the gauge $\mathbf{A}=B x \mathbf{e}_{y}$, the exact eigenstates and eigenvalues are

$$
\begin{gathered}
\Psi_{n k}=\left(2^{n+1} n ! \pi^{\frac{3}{2}} L\right)^{-\frac{1}{2}} e^{-i k y} e^{-\frac{1}{2}\left(\frac{x}{L}-\frac{\omega_{\mathrm{c}}}{\Omega} k L\right)^{2}} H_{n}\left(\frac{x}{L}-\frac{\omega_{\mathrm{c}}}{\Omega} k L\right) \\
E_{n k}=\hbar \Omega\left(n+\frac{1}{2}\right)+V\left(k L^{2}\right),
\end{gathered}
$$

where $\Omega^{2} \equiv \omega_{0}^{2}+\omega_{\mathrm{c}}^{2}, L^{2} \equiv \hbar / m \Omega$, and where the $V$ appearing in (3.3) refers to (3.1). The states $\Psi_{n k}$ are normalized as in Section II.

The exact equilibrium number density is given by

$$
\rho=\sum_{n} \int_{-\infty}^{\infty} d k f\left[\hbar \Omega\left(n+\frac{1}{2}\right)+V\left(k L^{2}\right)-\mu\right]\left|\Psi_{n k}\right|^{2},
$$

where $\mu$ is the chemical potential. Using (3.2), this may be written as

$$
\begin{aligned}
\rho=\frac{1}{2 \pi \ell^{2}}\left(1+\frac{\omega_{0}^{2}}{\omega_{\mathrm{c}}^{2}}\right) \sum_{n} & \left(2^{n} n ! \pi^{\frac{1}{2}}\right)^{-1} \int_{-\infty}^{\infty} d K f\left[\hbar \Omega\left(n+\frac{1}{2}\right)+\frac{\Omega^{2}}{\omega_{\mathrm{c}}^{2}} V(K L)-\mu\right] \\
& \times e^{-\left(\frac{x}{L}-K\right)^{2}} H_{n}^{2}\left(\frac{x}{L}-K\right),
\end{aligned}
$$

where $K \equiv k L \Omega / \omega_{\mathrm{c}}$. To facilitate comparison with our general expressions, we have written the prefactor in terms of the magnetic length $\ell$ rather than $L$.

In Fig. 1, we compare the exact ground-state density with the approximate distribution (1.3), for the case $\mu=3 \hbar \omega_{\mathrm{c}}$, where there are three Landau levels filled in the center of the 
well. The curvature of the confining potential is chosen to be $\omega_{0}=\frac{1}{20} \omega_{\mathrm{c}}$. The principal difference between the exact density profile (dashed curve) and the approximate profile (solid curve) is that the latter neglects the detail at the step edges. The actual density at the edges changes over a few magnetic lengths, and in a manner which depends on the particular Landau level involved.

There are two other differences between the density profiles, which are too small to be visible in Fig. 1. The first is that the density of a filled Landau level in the parabolically confined system is slightly greater than $\left(2 \pi \ell^{2}\right)^{-1}$, as is evident from the prefactor of (3.5). This reflects the small compressibility of the 2D electron gas at integer filling factors, which appears as a response to the second derivative of the confining potential, $V^{\prime \prime}(x)$, and which is neglected in (1.3). The second difference is that the length scale $L$ appearing in (3.5) is slightly less than the magnetic length $\ell$,

$$
L=\left(1-\frac{\omega_{0}^{2}}{4 \omega_{\mathrm{c}}^{2}}\right) \ell
$$

Hence, the actual density profile is slightly contracted relative to the profile given by (1.3).

The exact equilibrium current density, which is directed in the $y$ direction, may be written as

$$
j=\sum_{n} \int_{-\infty}^{\infty} d k f\left[\hbar \Omega\left(n+\frac{1}{2}\right)+V\left(k L^{2}\right)-\mu\right] j_{n k}
$$

where

$$
j_{n k} \equiv-\frac{e}{m} \operatorname{Re} \Psi_{n k}^{*}\left(-i \hbar \frac{\partial}{\partial y}+\frac{e}{c} \mathbf{A} \cdot \mathbf{e}_{y}\right) \Psi_{n k}
$$

is the contribution to the current density from the state $\Psi_{n k}$. From (3.2), we obtain $j_{n k}=e \omega_{c}\left(k \ell^{2}-x\right)\left|\Psi_{n k}\right|^{2}$, which may be rewritten as

$$
j_{n k}=\frac{e L \Omega^{2}}{\omega_{\mathrm{c}}}\left(K-\frac{x}{L}\right)\left|\Psi_{n k}\right|^{2}+e \omega_{\mathrm{c}} \ell^{2}\left(\frac{V^{\prime}(x)}{\hbar \omega_{\mathrm{c}}}\right)\left|\Psi_{n k}\right|^{2} .
$$

Therefore, the current distribution may be written as the sum of a bulk current and an 
edge current, where the bulk contribution is exactly

$$
j_{\mathrm{bulk}}=e \omega_{\mathrm{c}} \ell^{2}\left(\frac{V^{\prime}(x)}{\hbar \omega_{\mathrm{c}}}\right) \rho(x)
$$

and where

$$
\begin{gathered}
j_{\text {edge }}=\frac{e \omega_{\mathrm{c}}}{2 \pi \ell}\left(1+\frac{\omega_{0}^{2}}{\omega_{\mathrm{c}}^{2}}\right)^{\frac{7}{4}} \sum_{n}\left(2^{n} n ! \pi^{\frac{1}{2}}\right)^{-1} \int_{-\infty}^{\infty} d K f\left[\hbar \Omega\left(n+\frac{1}{2}\right)+\frac{\Omega^{2}}{\omega_{\mathrm{c}}^{2}} V(K L)-\mu\right] \\
\times\left(K-\frac{x}{L}\right) e^{-\left(K-\frac{x}{L}\right)^{2}} H_{n}^{2}\left(K-\frac{x}{L}\right) .
\end{gathered}
$$

In Fig. 2 we compare the exact ground-state current distribution (dashed curve) to the current distribution given in Section I (solid curve). The bulk contributions to the current density are nearly identical; they differ only in that the density in (1.2) and the exact density in (3.10) are slightly different, as shown in Fig. 1. The sharp steps in $\rho$ lead to the sharp zig-zag structure in the solid curve of Fig. 2. However, we see that the approximate edge current (1.1), which at zero temperature consists of a series of $\delta$-functions, does not capture the form present in the exact edge current (3.11). However, as we shall show, the edge current (1.1) correctly accounts for the net current associated with a given edge (the integrated edge current density), in accordance with the earlier assertion that our distributions correctly describe the large-scale features of the exact distributions.

To prove this, we define the integrated edge currents, $I_{n}^{+}$and $I_{n}^{-}$, associated with the two edges of the $n$th Landau level, one edge located to the right $(+)$ of the origin and the other located to the left $(-) . I_{n}^{+}$is defined by

$$
\begin{gathered}
I_{n}^{+} \equiv \frac{e \omega_{\mathrm{c}}}{2 \pi \ell}\left(1+\frac{\omega_{0}^{2}}{\omega_{\mathrm{c}}^{2}}\right)^{\frac{7}{4}}\left(2^{n} n ! \pi^{\frac{1}{2}}\right)^{-1} \int_{0}^{\infty} d x \int_{-\infty}^{\infty} d K f\left[\hbar \Omega\left(n+\frac{1}{2}\right)+\frac{\Omega^{2}}{\omega_{\mathrm{c}}^{2}} V(K L)-\mu\right] \\
\times\left(K-\frac{x}{L}\right) e^{-\left(K-\frac{x}{L}\right)^{2}} H_{n}^{2}\left(K-\frac{x}{L}\right) .
\end{gathered}
$$

The definition of $I_{n}^{-}$, identical to (3.12) except that the $x$ integration is from $-\infty$ to 0 , shows that $I_{n}^{-}=-I_{n}^{+}$. This means that the integrated currents carried along the two edges 
of each Landau level are of equal magnitude and are opposite in sign, as is well-known. In Appendix I, we show that the integrated ground-state edge currents are equal to

$$
I_{n}^{ \pm}=\mp\left(n+\frac{1}{2}\right) \frac{e \omega_{\mathrm{c}}}{2 \pi}\left(1+\frac{\omega_{0}^{2}}{\omega_{\mathrm{c}}^{2}}\right)^{\frac{3}{2}} .
$$

This result is also valid for low temperatures such that $k_{\mathrm{B}} T<<\hbar \omega_{\mathrm{c}}$. The integrated edge currents given by the distribution (1.1) are easily shown to be

$$
I_{n}^{ \pm}=\mp\left(n+\frac{1}{2}\right) \frac{e \omega_{\mathrm{c}}}{2 \pi},
$$

which is equal to (3.13), apart from small corrections of order $\omega_{0}^{2} / \omega_{\mathrm{c}}^{2}$.

Fig. 2 also demonstrates that the direction of the current oscillates with position. This striking feature is correctly accounted for in our general expression by noting that $\nabla \rho$ is antiparallel to $\nabla V$. These oscillations, which originate from the oscillations in the magnetization of the $2 \mathrm{D}$ electron gas as a function of filling factor, are a generic feature of the current distribution in a confined 2D electron gas. This is explained further in Appendix II. 


\section{APPLICATIONS}

A. Current distribution in a Hall bar

The general expression we have derived for the low-temperature current distribution in a 2D electron gas can be easily used with a self-consistent potential obtained by solving the Hartree, Hartree-Fock, or Kohn-Sham equations. We now apply our result to a stepped potential characteristic of the low-temperature self-consistent Hartree potential of a narrow gate-confined Hall bar. The Hall bar is assumed to lie along the $y$ direction, with a confining potential $V(x)$ and chemical potential as shown in Fig. 3. A uniform magnetic field is applied in the $z$ direction.

The confining potential we use is similar to that obtained classically by Chklovskii et $a l .{ }^{6}$ for a narrow gate-confined channel, and confirmed by self-consistent Hartree and Hartree-Fock calculations. ${ }^{9-12}$. However, we have approximated the potential by a series of linear potentials and we have included small slopes $\left(10^{-3} \hbar \omega_{\mathrm{c}} / \ell\right)$, on the plateaus and in the central region, to include, in a simple fashion, the effects of imperfect screening. These slopes are too small to be resolved in Fig 3. We shall show that this piecewise linear potential, which supports a low-temperature density distribution consisting of wide compressible edge channels and narrow incompressible strips, is sufficient to accurately characterize the low-temperature current distribution in a narrow Hall bar.

In Fig. 4, we plot the low-temperature $\left(k_{\mathrm{B}} T=0.002 \hbar \omega_{\mathrm{c}}\right)$ density and current distributions in the confining potential of Fig. 3. The large number of fractionally occupied states in the compressible edge regions leads to smooth edge profiles (solid curve). The edge channels occur in the plateaus of the potential, whereas the incompressible strips occur at the potential steps. The current distribution (dashed curve) consists of edge currents 
in the compressible regions and bulk currents in the incompressible regions. Small steps occur in the density (at $x= \pm 15 \ell$ ) and current density (at $x=0$ ) because of the sharp corners in our piecewise linear potential.

\section{B. Universal integrated currents}

In the ideal case of perfect compressibility and incompressibility, only one type of current contributes to a given region. We now show that, in this ideal case, the integrated currents in these regions are universal, independent of the size and position of the regions, and the geometry of the sample. It is easy to calculate total current carried in each edge channel and in each incompressible strip (we neglect incompressible strips at fractional filling factors). From (1.1), we see that the integrated edge current depends only on the net density change across the edge channel, which is $\left(2 \pi \ell^{2}\right)^{-1}$ at low temperatures, and on the index $n$ of the Landau states which form the edge. Therefore, an edge channel at the edge of the $n$th Landau level carries a current of magnitude

$$
I_{\text {edge }, n}=\left(n+\frac{1}{2}\right) \frac{e \omega_{\mathrm{c}}}{2 \pi},
$$

independent of the width and position of the edge channel. A central compressible region which supports no net density change across its boundaries, carries no integrated current. From (1.2), we see that the integrated bulk current in an incompressible strip with integral filling factor $\nu$ is simply

$$
I_{\mathrm{bulk}}=\nu \frac{e \omega_{\mathrm{c}}}{2 \pi}
$$

independent of the width and position of the strip. Fig. 4 also demonstrates the alternating pattern of the directions of the edge and bulk currents.

In nonideal cases, for example at higher temperature, both the edge and bulk components contribute simultaneously, and there are corrections to (4.1) and (4.2). However, the oscillations in the directions of the currents generally remains. 


\section{Quantized persistent currents}

As a final application of our result, we shall investigate the quantization of the persistent current (total azimuthal current through a radial cross section) in a quantum dot predicted recently by Avishai and Kohmoto ${ }^{24}$. Consider a system of noninteracting electrons in a slowly varying cylindrically symmetric potential $V(r)$ subjected to a uniform magnetic field $\mathbf{B}=B \mathbf{e}_{z}$. The quantum dot is assumed to be large enough so that there are many degenerate Landau states in the bulk. The ground-state radial density and current distributions will be similar to those in Figs. 1 and 2 (with $x$ acting as a radial coordinate), except that the central incompressible region will be larger and the bulk currents will vanish there because of the assumed flatness of the confining potential. Following Ref. 24, we shall calculate the integrated azimuthal current

$$
I=\int_{0}^{\infty} d r j(r)
$$

when the Fermi energy lies in a bulk Landau level. We shall initially assume, for simplicity, that the Fermi energy is just below Landau level $n$ so that the filling factor is $\nu=n$ in the center of the dot. Afterwards, we treat the realistic situation where the Fermi energy is somewhere in the bulk states of Landau level $n$.

We shall evaluate the persistent current by dividing the integral (4.3) into $n$ regions of filling factor $n, n-1, \ldots, 1$. The central region is from $r=0$ (where the azimuthal current density vanishes) to $r=r^{\prime}$ where $V\left(r^{\prime}\right)-V(0)=\hbar \omega_{c}$, and has constant filling factor $\nu=n$. The integrated bulk current in this region is simply $n e \omega_{c} / 2 \pi$. The edge current concentrated about $r=r^{\prime}$ comes from states with Landau level index $n-1$ and contributes an amount $-\left(n-\frac{1}{2}\right) e \omega_{c} / 2 \pi$ to $(4.3)$. The integrated current in this first region is therefore $e \omega_{c} / 4 \pi$, independent of $n$. Beyond $r=r^{\prime}$, the filling factor decreases to $\nu=n-1, n-2, \ldots, 0$. It is simple to verify that the contribution to (4.3) from each of these regions is $e \omega_{c} / 4 \pi$. 
Therefore, whenever the Fermi energy lies just below the bulk states of Landau level $n$, the persistent current is $n e \omega_{c} / 4 \pi$.

As the number of electrons in the quantum dot is changed, the Fermi energy is generally pinned in a set of bulk states, not below them. Because the bulk states carry no bulk current, the persistent current calculated above is modified by the integrated edge current $-\left(n+\frac{1}{2}\right) e \omega_{c} / 2 \pi$ of Landau level $n$ only. Therefore, when the Fermi energy is locked in the bulk states of Landau level $n$, the persistent current (4.3) is

$$
I=-(n+1) \frac{e \omega_{c}}{4 \pi}
$$

Avishai and Kohmoto ${ }^{24}$ have also predicted a persitent current which is quantized in integer multiples of $e \omega_{c} / 4 \pi$ as a function of particle number.

\section{ACKNOWLEDGMENTS}

This work was supported by the National Science Foundation through Grant No. DMR-9100988. We thank Rolf Gerhardts, Yigal Meir, Boris Shklovskii, and David Thouless, for providing us with preprints of their work. 


\section{APPENDIX I}

Here we calculate the integrated ground-state edge currents, $I_{n}^{+}$, which may be written as

$$
\begin{aligned}
I_{n}^{+} \equiv g A_{n} \int_{0}^{\infty} d x \int_{-\infty}^{\infty} d K \Theta\left[\mu-\hbar \Omega\left(n+\frac{1}{2}\right)-\frac{\Omega^{2}}{\omega_{\mathrm{c}}^{2}} V(K L)\right] \\
\times\left(K-\frac{x}{L}\right) e^{-\left(K-\frac{x}{L}\right)^{2}} H_{n}^{2}\left(K-\frac{x}{L}\right)
\end{aligned}
$$

where $A_{n} \equiv\left(2^{n} n ! \pi^{\frac{1}{2}}\right)^{-1}, g \equiv\left(e \omega_{\mathrm{c}} / 2 \pi \ell\right)\left[1+\left(\omega_{0} / \omega_{\mathrm{c}}\right)^{2}\right]^{\frac{7}{4}}$, and where $\Theta[\epsilon]$ is the unit step function. The Hermite polynomial relations $x H_{n}=\frac{1}{2} H_{n+1}+n H_{n-1}$ and $H_{n}^{\prime}=2 n H_{n-1}$ lead to

$$
\begin{gathered}
I_{n}^{+}=-\frac{1}{2} g L A_{n} \int_{0}^{\infty} d X \int_{-K_{n}}^{K_{n}} d K e^{-(X-K)^{2}} \\
\times H_{n}(X-K)\left[H_{n+1}(X-K)+\frac{\partial}{\partial X} H_{n}(X-K)\right],
\end{gathered}
$$

where $X \equiv x / L$, and where $K_{n} L$, defined by $\mu=\hbar \Omega\left(n+\frac{1}{2}\right)+\left(\Omega^{2} / \omega_{\mathrm{c}}^{2}\right) V\left(K_{n} L\right)$, is the center of the $n$th edge. By using $H_{n}=\frac{1}{2} H_{n+1}^{\prime} /(n+1)$, we find

$$
\begin{aligned}
I_{n}^{+} & =-\frac{1}{4} g L A_{n} \int_{0}^{\infty} d X \int_{-K_{n}}^{K_{n}} d K e^{-(X-K)^{2}} \\
& \times \frac{\partial}{\partial X}\left(\frac{H_{n+1}^{2}(X-K)}{2(n+1)}+H_{n}^{2}(X-K)\right) .
\end{aligned}
$$

After integrating by parts, assuming that $K_{n}>>1$ (which is equivalent to $\omega_{0}<<\omega_{\mathrm{c}}$ ), we obtain the recurrence relation $I_{n+1}^{+}=I_{n}^{+}-g L$, which leads to $I_{n}^{+}=I_{0}^{+}-n g L$. From (A1), we find $I_{0}^{+}=-\frac{1}{2} g L$. Therefore, the zero-temperature integrated edge currents are equal to

$$
I_{n}^{ \pm}=\mp\left(n+\frac{1}{2}\right) \frac{e \omega_{\mathrm{c}}}{2 \pi}\left(1+\frac{\omega_{0}^{2}}{\omega_{\mathrm{c}}^{2}}\right)^{\frac{3}{2}}
$$

as stated. 


\section{APPENDIX II}

In this appendix, we shall derive the current distribution given in Section I from linear response theory to provide a deeper understanding of the result and to establish the connection with the distribution derived from current-density functional theory. ${ }^{1}$ We start with a uniform electron gas in a magnetic field $\mathbf{B}=B \mathbf{e}_{z}$ at low temperature $\left(k_{\mathrm{B}} T<<\hbar \omega_{\mathrm{c}}\right)$. The static current-current response function $\chi^{\mu \nu}(\mathbf{q})(\mu, \nu=0,1,2)$, defined by $j^{\mu}(\mathbf{q})=\chi^{\mu \nu}(\mathbf{q}) A^{\nu}(\mathbf{q})$, describes the Fourier components of the three-current $j^{\mu} \equiv(\rho, \mathbf{j})$ induced by the application of an infinitesimal potential $A^{\mu} \equiv(V, \mathbf{A})$. Combining $\chi^{00}(\mathbf{q})$ and $\chi^{i 0}(\mathbf{q})$ together leads to the nonlocal relation

$$
j^{i}(\mathbf{q})=\frac{\chi^{i 0}(\mathbf{q})}{\chi^{00}(\mathbf{q})} \rho(\mathbf{q})
$$

between current and density. It is simple to show that $\chi^{i 0}=-i c\left(\mathbf{q} \times \mathbf{e}_{z}\right)^{i}(\partial M / \partial \mu)_{B}$ and $\chi^{00}=-(\partial \rho / \partial \mu)_{B}$ in the long wavelength limit, where $M$ is the orbital magnetization of a uniform $2 \mathrm{D}$ electron gas. The Maxwell relation $(\partial M / \partial \mu)_{B}=(\partial \rho / \partial B)_{\mu}$ then leads to $\mathbf{j}(\mathbf{r})=-(e \hbar / 2 m) \gamma \nabla \rho(\mathbf{r}) \times \mathbf{e}_{z}$, where

$$
\gamma \equiv-\frac{2 m c}{e \hbar} \frac{(\partial \rho / \partial B)_{\mu}}{(\partial \rho / \partial \mu)_{B}} .
$$

This is equivalent to the LDA result derived in Ref. 1.

In the uniform electron gas,

$$
\left(\frac{\partial \rho}{\partial B}\right)_{\mu}=\frac{\rho}{B}-\frac{1}{2 \pi \ell^{2}}\left(\frac{e \hbar}{m c}\right) \sum_{n}\left(n+\frac{1}{2}\right) F_{n}^{-1}(\mu)
$$

and

$$
\left(\frac{\partial \rho}{\partial \mu}\right)_{B}=\frac{1}{2 \pi \ell^{2}} \sum_{n} F_{n}^{-1}(\mu)
$$

where $F_{n}(\mu) \equiv 4 k_{\mathrm{B}} T \cosh ^{2}\left(\left[\mu-\hbar \omega_{\mathrm{c}}\left(n+\frac{1}{2}\right)\right] / 2 k_{\mathrm{B}} T\right)$. At low temperatures, $F_{n}^{-1}(\mu)$ is strongly localized about $\mu=\hbar \omega_{\mathrm{c}}\left(n+\frac{1}{2}\right)$ with a width of order $k_{\mathrm{B}} T$. Note that the 
denominator in (B2) is positive definite, whereas the numerator is a sum of two terms with opposite signs. We shall show that the first term in (B3) largely determines the current density in the incompressible regions while the second term largely determines the current in the compressible regions.

In a compressible region near filling factor $\nu=n+\frac{1}{2}$, the chemical potential is $\mu \approx \hbar \omega_{\mathrm{c}}\left(n+\frac{1}{2}\right)$. The narrow range $\left|\mu-\hbar \omega_{\mathrm{c}}\right| \leq k_{\mathrm{B}} T$ corresponds to the entire range of compressible densities. Because only a single term contributes to the summations (apart from terms exponentially small in $\left.k_{\mathrm{B}} T / \hbar \omega_{\mathrm{c}}\right)$,

$$
\gamma=-\frac{2 \rho}{\hbar \omega_{\mathrm{c}}}\left(\frac{\partial \rho}{\partial \mu}\right)_{B}^{-1}+(2 n+1)
$$

Then $\nabla \rho=-(\partial \rho / \partial \mu)_{B} \nabla V$ leads to the distribution given in (1.1) and (1.2), at filling factor $\nu=n+\frac{1}{2}$. The current density in a compressible region, where the self-consistent potential is nearly uniform, is largely determined by the second term in (B5). In an incompressible region, for example near $\nu=n$, the $F_{n}$ terms vanish with exponential accuracy, and the bulk term (1.2) dominates.

By construction, linear response theory is valid only when the perturbing stimulus is infinitesimal. However, the results obtained in this appendix are apparently valid even when the applied potential $V(\mathbf{r})$ is large, as long as $V(\mathbf{r})$ is slowly varying so that the long wavelength limit applies. We speculate that the reason for this is that the response function $\chi^{\mu \nu}\left(\mathbf{r}, \mathbf{r}^{\prime}\right)$, a correlation function of the three-currents $j^{\mu}(\mathbf{r})$ and $j^{\nu}\left(\mathbf{r}^{\prime}\right)$, is short-ranged in $\left|\mathbf{r}-\mathbf{r}^{\prime}\right|$. Then, as we have demonstrated with the single-particle Green's function, the effect of the confining potential may be treated locally as a shift in the chemical potential, plus the linear response of a uniform electron gas with this shifted chemical potential to a weak electric field. 


\section{REFERENCES}

1 G. Vignale and P. Skudlarski, Phys. Rev. B 46, 10232 (1992). See also G. Vignale, in Proceedings of the NATO ASI on Density Functional Theory, edited by E. K. U. Gross and R. M. Dreizler (in press).

2 G. Vignale and M. Rasolt, Phys. Rev. Lett. 59, 2360 (1987); Phys. Rev. B 37, 10685 (1988).

3 C. W. J. Beenakker and H. van Houten, in Solid State Physics Vol. 44, edited by H. Ehrenreich and D. Turnbull (Academic Press, New York, 1991).

${ }^{4}$ M. Büttiker, in Semiconductors and Semimetals Vol. 35, edited by M. Reed (Academic Press, New York, 1992).

${ }^{5}$ D. B. Chklovskii, B. I. Shklovskii, and L. I. Glazman, Phys. Rev. B 46, 4026 (1992).

6 P. L. McEuen et al., Phys. Rev B 45, 11419 (1992).

7 D. B. Chklovskii, K. A. Matveev, and B. I. Shklovskii, Phys. Rev. B 47, 12605 (1993).

8 M. M. Fogler, E. I. Levin, and B. I. Shklovskii (to be published).

9 B. Y. Gelfand and B. I. Halperin, Phys. Rev. B 49, 1862 (1994).

${ }^{10}$ L. Brey, J. J. Palacios, and C. Tejedor, Phys. Rev. B 47, 13884 (1993).

11 C. Wexler and D. J. Thouless, Phys. Rev. B 49, 4815 (1994).

12 K. Lier and R. R. Gerhardts, (to be published).

13 C. de C. Chamon and X. G. Wen, Phys. Rev. B 49, 8227 (1994).

14 Y. Meir, Phys. Rev. Lett. 72, 2624 (1994). 
15 A. H. MacDonald, T. M. Rice, and W. F. Brinkman, Phys. Rev. B 28, 3648 (1983).

16 O. Heinonen and P. L. Taylor, Phys. Rev. B 32, 633 (1985).

17 D. J. Thouless, J. Phys. C 18, 6211 (1985).

18 Q. Li and D. J. Thouless, Phys. Rev. Lett. 65, 767 (1990).

19 D. Pfannkuche and J. Hajdu, Phys. Rev. B 46, 7032 (1992).

20 D. J. Thouless, Phys. Rev. Lett. 71, 1879 (1993).

21 O. Heinonen and M. D. Johnson, Phys. Rev Lett. 71, 1447 (1993).

22 T. K. Ng, Phys. Rev. Lett. 68, 1018 (1992).

23 S. Hershfield, Phys. Rev. Lett. 70, 2134 (1993).

24 Y. Avishai and M. Kohmoto, Phys. Rev. Lett. 71, 279 (1993). 


\section{FIGURE CAPTIONS}

FIG. 1. Ground-state density, $\rho(x)$, in a parabolic potential with curvature $\omega_{0}=\frac{1}{20} \omega_{\mathrm{c}}$ and chemical potential $\mu=3 \hbar \omega_{\mathrm{c}}$, plotted in units of $\rho_{0} \equiv\left(2 \pi \ell^{2}\right)^{-1}$. The solid curve follows from the expression (1.3) in the text; the dashed curve is exact. Lengths are plotted in units of magnetic length $\ell$.

FIG. 2. Exact and approximate $y$ components of the ground-state current density, $j(x)$, in a parabolic potential, with the same parameters as in Fig. 1. The current distributions are plotted in units of $j_{0} \equiv e \omega_{\mathrm{c}} / 2 \pi \ell$. The solid curve follows from the distribution of Section I, with the vertical arrows denoting $\delta$-functions. The dashed curve is exact. Lengths are plotted in units of magnetic length $\ell$. Note the alternating directions, or signs, of the edge and bulk currents.

FIG. 3. Piecewise linear confining potential (solid curve) characteristic of the lowtemperature self-consistent Hartree potential of a narrow gate-confined Hall bar. The potential is plotted in units of $\hbar \omega_{\mathrm{c}}$, and the dashed line is the chemical potential $\mu=\frac{3}{2} \hbar \omega_{\text {c }}$. Lengths are plotted in units of magnetic length $\ell$.

FIG. 4. Equilibrium density (solid curve) and current density (dashed curve) corresponding to the stepped confining potential and chemical potential of Fig. 3, at low

temperature $\left(k_{\mathrm{B}} T=0.002 \hbar \omega_{\mathrm{C}}\right)$. The density is plotted in units of $\rho_{0} \equiv\left(2 \pi \ell^{2}\right)^{-1}$ and 
the current density is plotted in units of $j_{0} \equiv e \omega_{\mathrm{c}} / 2 \pi \ell$. Lengths are plotted in units of magnetic length $\ell$. Note the alternating directions, or signs, of the edge and bulk currents. 